\title{
Impfungen - viele Fragen, unterschiedliche Einschätzungen
}

\author{
Replik der Stiftung für Konsumentenschutz SKS auf die in der Schweizerischen Ärztezeitung Nr. 9/2005 \\ von der Eidgenössischen Kommission für Impffragen unter der Federführung von Prof. Dr. med. C.-A. Siegrist \\ vorgebrachte Kritik am Ratgeber der SKS «Impfen: Grundlagen für einen persönlichen Impfentscheid»
}

\footnotetext{
a Ständerätin, Präsidentin der Stiftung für Konsumentenschutz

b Geschäftsführerin der Stiftung für Konsumentenschutz
}

Korrespondenz: Stiftung für Konsumentenschutz Monbijoustrasse 61 Postfach

CH-3000 Bern 23

Tel. 0313702424

Fax 0313720027

E-Mail:

admin@konsumentenschutz.ch
«Eines ist mir aufgefallen: in der Impfdebatte gibt es zu vielen Fragen sehr unterschiedliche Einschätzungen...», schrieb Dr. Christoph RehmannSutter, Präsident der Nationalen Ethik-Kommission im Bereich Humanmedizin unlängst unter dem Titel «Die Impfdebatte - Streit um den Sinn der Medizin». Und er folgert daraus, dass es eine ethische Aufgabe sei, die Eltern bei ihren Entscheidungen zu unterstützen, indem man sie möglichst breit über alle Fakten informiere.

Mit dem Ratgeber «Impfen - Grundlagen für einen persönlichen Impfentscheid» leistet die Stiftung für Konsumentenschutz hierzu einen Beitrag. Eine ihrer zentralen Aufgaben besteht nämlich darin, den Konsumentinnen und Konsumenten unabhängige, nicht von wirtschaftlichen Interessen geleitete Informationen zur Verfügung zu stellen. Die Konsumentenschaft soll dadurch in die Lage versetzt werden, informierte Entscheide zu fällen.

Erstaunt nehmen wir zur Kenntnis, dass unser Ratgeber über das Impfen bei den Mitgliedern der Eidg. Impfkommission offenbar zu Verärgerung geführt und eine massive Polemik ausgelöst hat. Besonders ungehalten scheint man darüber zu sein, dass eine angesehene Stiftung wie die SKS einen Ratgeber zum Thema Impfen publiziert.

Wir sind aber auch darüber erstaunt, dass sich die Eidg. Impfkommission erst heute öffentlich zu Wort meldet. Der Impfratgeber der SKS wurde nämlich vor fünf Jahren erstmals publiziert und erreichte im Februar 2004 aufgrund der grossen Nachfrage bereits die fünfte Auflage.

Die Gründe, weshalb sich die SKS vor fünf Jahren entschlossen hat, einen Ratgeber zum Thema Impfen herauszugeben, listet Frau Siegrist im Interview gleich selber auf: Während Jahren wurden nicht nur Ärztinnen und Ärzte, sondern auch Eltern von offizieller Seite her sehr einseitig und unausgewogen informiert. Dies hatte zur Folge, dass sich viele Eltern angesichts des Drohfingers der Gesundheitsbehörden unter Druck gesetzt fühlten. Sie waren auf der Suche nach zusätzlichen, ergänzenden und differenzierten Informationen zum Thema Impfen. Diesem Bedürfnis kam die SKS mit dem Impfratgeber nach.

Wenn sich der Kinderarzt nun bei uns beklagt, dass er wegen unseres Ratgebers vor dem Impfen noch eine Beratung durchführen müsse, dann haben wir dafür wenig Verständnis. Und wenn Frau Siegrist sich darüber beklagt, dass unser Ratgeber Zweifel säe und damit das Vertrauen der Patienten in ihren Arzt gefährde, dann scheint dieses Arzt-Patienten-Verhältnis, das so rasch erschüttert ist, ohnehin auf schwachen Füssen zu stehen.

Beim Impfen müssen Eltern für ihr Kind wichtige Entscheide fällen. Da darf das Stellen von Fragen nicht bereits ein Misstrauensvotum gegenüber der Ärztin, dem Arzt sein. Wir sind überzeugt, dass Patientinnen und Patienten ihr Vertrauen zu einem Arzt aufbauen, der ihre Bedenken ernst nimmt und sie mit guten, fachlichen Argumenten überzeugt. Nicht blindes Vertrauen ist unser Ziel, sondern ein partnerschaftliches Verhältnis.

Mit den Informationen, welche im Impfratgeber enthalten sind, wollen wir weder «die Impfstoffe, die Impfprogramme, die Gesundheitsbehörden und die sie anwendenden Ärzte in Verruf bringen» (Zitat Siegrist), noch fassen wir die Broschüre als Angriff auf das BAG auf. Wir wollen auch nicht gegen das Impfen «in den Krieg ziehen». Dieses für die Kritik an unserem Impfratgeber verwendete Vokabular zeigt, wie schwierig ein Dialog ist.

Eine konstruktive Auseinandersetzung mit der Impfthematik setzt eine differenzierte Information voraus. Gerade diese wird aber von vielen Eltern vermisst. So erhalten alle jungen Eltern zum Beispiel einen kostenlosen Ratgeber (Present Service «baby Ratgeber und Begleiter für junge Eltern - das erste Lebensjahr»), der in einer jährlichen Auflage von 60000 bis 70000 Exemplaren gedruckt wird. Der Ratgeber stellt auf 
drastische Weise die Folgen des Nicht-Impfens dar. Allfällige Nebenwirkungen des Impfens hingegen werden mit keinem Wort erwähnt.

Die SKS hat sich im August 2003 mit dem BAG intensiv über den Impfratgeber auseinandergesetzt. Die vom BAG beanstandeten Stellen im Ratgeber wurden entsprechend angepasst. In einem abschliessenden Gespräch mit der SKS hat das BAG festgehalten, dass eine kritische Beurteilung der Impfempfehlungen in Ordnung sei und vom BAG begrüsst werde. Auf eine von Prof. C. Aebi im August 2004 vorgebrachte Kritik haben die Autoren des Ratgebers ebenfalls sorgfältig entgegnet. Von Prof. Aebi kamen keine weiteren Rückmeldungen.

Die Stiftung für Konsumentenschutz steht vollumfänglich hinter den Autoren des Ratgebers. Ihre differenzierte Art und Weise, das Für und Wider sowie den allenfalls richtigen Zeitpunkt einer Impfung abzuwägen, hat auch Tausende von Leserinnen und Lesern überzeugt.

Die Autoren Dr. med. Martin Hirte, Facharzt für Kinderheilkunde/Allergologie, und Dr. med. Hansueli Albonico, FMH Allgemeinmedizin, haben eine Replik auf die Detailkritik verfasst, welche der Chefredaktion der Ärztezeitung vorliegt. Es wurde uns zugesichert, dass diese Replik in einer der nächsten Ausgaben publiziert wird. Wir danken der Schweizerischen Ärztezeitung für diese Möglichkeit zur Stellungnahme, denn die Sachlage rund ums Impfen ist keineswegs derart eindeutig und unproblematisch, wie das die EKIF glauben machen will. Die Replik ist ab dem Zeitpunkt der Veröffentlichung in der Ärztezeitung ebenfalls auf www.konsumenten schutz.ch verfügbar. 